\title{
Feasibility of Desktop Virtualization Per Software Services and Local Hardware Based on the Network Throughput
}

\author{
Vitor Chaves De Oliveira, Inacio Henrique Yano, \\ Alexandre De Assis Mota and Lia Toledo Moreira Mota \\ Department of CEATEC, Faculty of Electrical Engineering, \\ University of Pontifical Catholic, Campinas-São Paulo, CEP 13086-900, Brazil
}

Received 2013-05-08, Revised 2013-05-24; Accepted 2013-07-05

\begin{abstract}
In recent years, virtualization computing has become a worldwide reality present in datacenters servers of most organizations. The motivations for the use of this solution are focused primarily on cost reduction and increases in availability, integrity and security of data. Based on these benefits, recently it was started the use of this technology for personal computers as well. That is, for desktops, giving birth to the so-called desktop virtualization. Given the technical advantages of the approach, its growth has been so significant that, before 2014, it is expected to be present in over $90 \%$ of organizations. However, this new method is completely based on a physical client-server architecture, which increases the importance of the communication network that makes this technique possible. Therefore, analyzing the network in order to investigate the effects according to the environment implemented becomes crucial. In this study it's varied the local's client hardware and the application, i.e., the service used. The purpose was to detail their effects on computer networks in a Quality of Service (QoS) parameter, throughput. Secondarily are outlined perceptions regarding the Quality of Experience (QoE)? This culminated in an assessment that traces the feasibility for applying this technology.
\end{abstract}

Keywords: Desktop Virtualization, Computer Networks, Quality of Service (QoS), Quality of Experience (QoE), Client-Server Architecture, Thin Client, Electric Energy Consumption

\section{INTRODUCTION}

Given the rapid pace at which the Desktop Virtualization technique is being disseminated (CITRIX, 2011; Thibodeau, 2012; Oliveira et al., 2013), it is of fundamental importance to study its effects. Since, to design a network to support such solutions, metrics are necessary to outline their impacts. In this study, such study was performed through controlled trials that helped to analyze some characteristics of the Virtual Desktop Infraestructure (VDI), including the disadvantages associated with this technique. Because, once its benefits are already well-established and well-known (Eden, 2011), the analysis of the results from this study, which also includes the disadvantages of the technique, plays a role of significative relevance.

In this study, it was investigated how the traffic in networks with desktop virtualization is affected by different uses of local hardware and software services. Specifically, it was measured a Quality of Service (QoS) parameter in computer networks with VDI for distinct and classifiable experimental setups. This parameter was the flow rate, i.e., throughput (Mota et al., 2011; GCNET, 2012; Adibi et al., 2010). Such Corresponding Author: Vitor Chaves De Oliveira, Department of CEATEC, Faculty of Electrical Engineering, University of Pontifical Catholic, Campinas-São Paulo, CEP 13086-900, Brazil 
implementations have addressed how the reduction in local physical hardware of the access client can be applied to each service tested, as well as its cost on the communication channel. Thus, this study seeks to establish how the relationships between application, employed access client and network traffic work.

Second airily, perceptions are exposed regarding the Quality of Experience for each setup balancing it with the measured throughput. That is, for each hardware implementation along with the use of a particular software tool, it was correlated to the measure of the QoS parameter with the degree of satisfaction of the user experience. As the QoE definition delimits the level of user satisfaction, a relevant property to the data captured from the network is obtained. Therefore, it consolidates the knowledge on the impacts of the solution that is the subject of this study.

Finally, this study provides information for the design of networks with virtual desktop infrastructure, considering issues related to management techniques. Such data should, consequently, provide that decisions about the adoption of solutions of this nature have all its cost taken into consideration.

This study addresses the implications of changes in local hardware and application variation on the throughput of computers networks with desktop virtualization. Applications are classified into four software services: Video, Audio, Text and Navigation (Web Browser). The variation of the physical hardware in the access client was characterized by two devices: Thin Client-TC ('Slim' Terminal) and Fat Client-FC ('Robust' Terminal). As for the physical server used in the tests, it is noteworthy that there is the use of a structure of a server composed by a high performance personal computer and it is utilized for the experiments performed in all setups proposed. The communication channel, in which the studies were conducted, was the Ethernet networks, also known as IEEE 802.3 (IEEEInstitute of Electrical and Electronic Engineers).

\section{MATERIALS AND METHODS}

The structure of the equipment utilized in the experimental set-'Setups' is outlined in Fig. 1 below. This structure is called, for the sake of convenience, physical structure, while the other compositions that complete the setup, each one allowing the differentiation of equipment, software service and

The logical structure was defined with the following naming scheme in order to make it to be intuitive and self-explanatory for each Setup. Thus, each setup is defined by.

\section{Access Client + Computational peration + Software} Service.

Examples:

- FatClient_Acessing_Physical_Desktop_Video

- ThinClient_Streaming_Audio

- ThinClient Acessing Virtual Desktop Text

- FatClient_Acessing_Virtual_Desktop_WebBrowser

As an example, suppose it is wanted to analyze the 'lean' client for the implementation of virtualized desktop testing playback of the video file. Such a setup would be defined by: “ThinClient_Acessing_Virtual_Desktop_Video".

\subsection{Network Traffic Analyzer-Sniffer}

In the investigation carried out in this study, the role of the network traffic analyzer, i.e., Sniffer is crucial because it is through it that all measurements of quality of service parameters of a network are recorded. This tool is able to intercept and examine all the data packets that transit in a given communication channel. This identification allows capturing specific information, parted or congregated by configurable predetermined characteristics that it is demanded to observe and examine. It is emphasized that a network traffic analyzer can be either a hardware device designed or adapted for this purpose, as well as a computer software program.

In this study, the sniffer used to monitor QoS parameters was the WireShark Network Protocol Analyzer ${ }^{\circledR}$ Version 1.7.0. It is noteworthy that this computer program is free open source software, i.e., free access, modification and distribution. Thus, WireShark contitutes a programmable and/or adaptable to whatever necessity or requirement. Another important feature of WireShark is that it does not have an active role in the network, not placing, removing and/or altering any traffic. This means that its only function is related to the channel (i.e., 'network') monitoring WireShark Foundation, 2012.

\subsection{Server's Physical Machine-High Performance Personal Computer}

Manufacturer: Dell, Model: Dell® Vostro 260s Slim, Integrated Network Card: 10/100/1000 Ethernet LAN on system board; Memory: 6 GB DDR3 SDRAM at 1333 $\mathrm{MHz}$, Processor: 2nd Generation Intel ${ }^{\circledR}$ Core Tм i52400 (3.1GHz até 3.4GHz-Turbo Boost 2.0, 4 Threads, $6 \mathrm{Mb}$ Cache, 64 bits), Video Card: AMD Radeon HD 64501GB DDR3, Dimensions (width, height and depth): $35.0 \times 10.5 \times 45.0 \mathrm{~cm}$. 


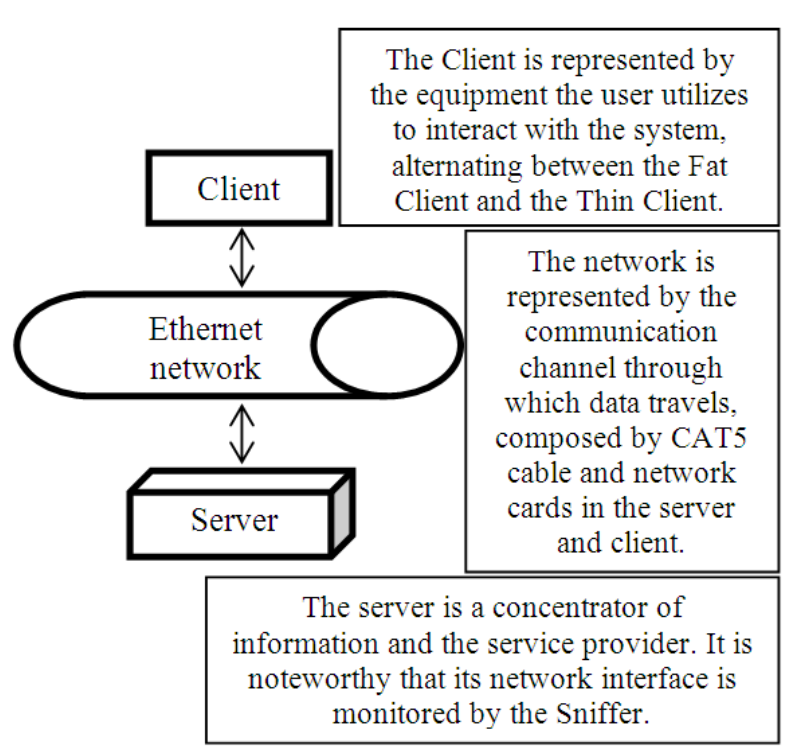

Fig. 1. Setups physical structure

Other relevant information pertinent to the server:

- Operating System installed on the physical machine: Server: Microsoft Windows Server ${ }^{\circledR} 2008$ R2 Enterprise (64bits)

- Software to Monitor Network Interface: Installed on the server: WireShark Network Protocol Analyzer ${ }^{\circledR}$ Version 1.7.0

- Hypervisor Software: Installed on Server: VMware Workstation ${ }^{\circledR} 8$

- Virtualized Hardware (created from the Hypervisor): Processors: 4, Memory: 4GB, HDD: 60GB, Network Card: Ethernet

- Operating System Installed on the Virtual Machine: Microsoft ${ }^{\circledR}$ Windows 7 Ultimate (64bits)

- Software for Media Playback-installed on all OS of this experiment, except the Thin Client's OS: VLC Media Player 2.0.1

- $\quad$ Software for Text: Notepad (Windows native)

- $\quad$ Software for Navigation: Mozilla Firefox ${ }^{\circledR}$

\subsection{Client's Physical Machine-Thin Client Access Terminal}

Manufacturer: Net Computer Technology Co. Ltd., Model: NC600W, Integrated Network Card: 10/100/1000 Ethernet LAN on system board, Memory: 20MB RAM, processor: $533 \mathrm{Mhz}$, native OS: Microsoft Windows ${ }^{\circledR}$ CE 5.0, Dimensions (width, height and depth): $11.9 \times 11.9 \times 2.5 \mathrm{~cm}$.

\subsection{Client's Physical Machine-High Performance Access Terminal (Fat Client)}

Manufacturer: Dell, Model: Dell Alienware M11x Laptop, Integrated Network Card: 10/100/1000 Ethernet LAN on system board, Memory: 6 GB DDR3 SDRAM at $1333 \mathrm{MHz}$, Processor: 2nd Generation Intel ${ }^{\circledR}$ Core TM i7-2637M (1.7GHz, 4 Threads, 4Mb Cache) OS: Microsoft Windows 7 Home Basic (64bits) ®; Dimensions (width, height and depth) $28.5 \times 2.3 \times 22.0 \mathrm{~cm}$.

\subsubsection{Transmission Channel-Ethernet Network IEEE 802.3}

Figure 2 illustrates the point-to-point Ethernet Network present in the experiments. This is a schematic of all the physical elements present in the network in question. That is, there is the diagram showing the ethernet network board (card) of client and server and network cable CAT5e RJ45 connectors to the server and the client. All these elements compose the network, this is the transmission channel.

\subsection{Detailing the Study}

The setup of the investigations, that is, the assembly of the environment, consisted in connecting, alternately, the server to the clients and monitor traffic on the physical network interface located in the server. A Fig. 1 and 2 illustrate this. It can be observed that the server is, at one time connected to the Thin Client and at another time connected to the Fat Client. In total, there were five different computational operations conducted, described as follows.

The stages that tested the audio and video services occurred as follows. In the first case, understood as the case of employment of local conventional structure, occurred the local client capacity utilization. i.e., the fat clients via remote access file or stream media ('streaming'), performing only one transmission of video or audio, using the local structure. For this, the server is connected to a Fat Client and through the program VLC Media Player, installed on both server OS, as the OS of the Fat client, originated media playback. This file multimedia content was in Server's Hard Drive and was accessed by the client, which initiated the transmission. The moment that the playback started, monitoring the network interface of the server via WireShark sniffer software, also started. Importantly, these tests were conducted producing the file with the collections of information in segmented sections sixty seconds. In the second case, referred to as a session of the physical environment with Fat Client, also understood by Desktop Access to Physical Server through 'Robust' Client. 


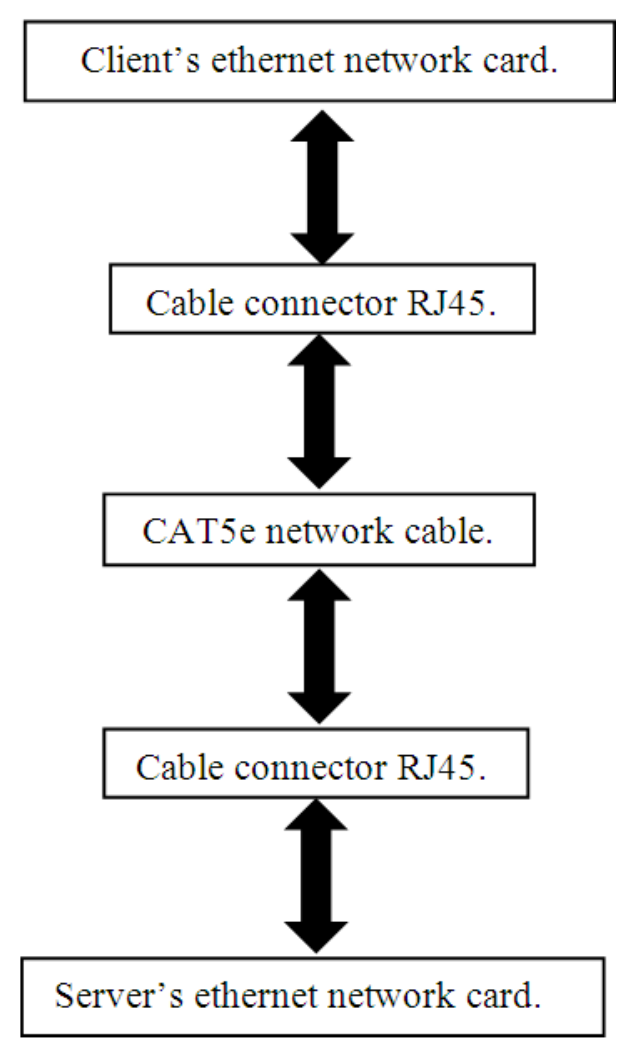

Fig. 2. Point-to-point ethernet network

It was carried out a transmission of a session via Remote Desktop Protocol (RDP) of the OS installed on the Server, reproducing the same files (audio and video). It is noteworthy that in this trial, the file itself was not physically transmitted from one machine to another, as in the previous case, but played on a machine (server) and displayed in another (Fat Client). The same happened with the third evaluation, called Server's Physical Desktop Access with a 'Slim' Terminal, the difference, for this case, is that the access client changed to Thin Client. The last two cases were achieved through the prism of the desktop virtualization technology. Such an environment has been implemented as follows: on the Server's OS is installed a program that enables the creation of virtual hardware, the hypervisor, called VMW are WorkStation. With this software, it is created a virtual machine, whose resources have already been explained above. On top of this virtual machine, it is installed an OS and that created a Virtual Desktop (VD). In this VD, it was placed the referred files to be displayed or the service to run. Thus, the service in question was located on the hard disk of the VD. The
Virtual Desktop is accessed by clients in a session via RDP protocol and the tool present in it was played to the client's screen.

As for the text service, it was performed a writing ('typing') test the loked ('triggered') the " $A$ " key, in uppercase, with the same character repetition speed on all keypads for all examinations. Such speed was set at exactly the intermediate value between average speed and maximum speed. In this service, it is worth to point out that there is no way to measure the use, in relation to the monitoring network, a traditional local structure. This is because this service only exists in a local and conventional solutions, it does not depend on a communication network to be executed. Therefore, tests were done only with a remote access (via RDP) to the physical and virtual desktop through robust client and thin client.

For the navigation service, the same characteristic of not having the measurement of a conventional local structure is present. The reason for this is that the goal of this study is to measure the cost of VDI and this cost, in this case, only exists between a client and a server through the access in a remote session of a Physical or Virtual Desktop via a robust client and thin client. Furthermore, it is necessary to characterize the navigation service experiments, since it was conducted with certain characteristics. The first step before conducting the tests was the local retrieving ('saving') and previously loading in the machines OSs (Physical and Virtual) of ten random web pages. These pages contained mixed content, as they are known in the Brazilian Internet portals, including: Brazil Yahoo, Google Brazil, Carta Capital, UOL, Globo.com, Google Earth, Microsoft. These pages were saved and preloaded ('open') in tabs in a single window in the Firefox Web Browser. Only with this completed, it is initiated manually and randomly testing this service for each experimental characterized setup.

It is emphasized that the tests with the different clients, Thin Client and Fat Client occurred separately. Thus, firstly, a setup is accessed it with one type of client, the tests are ran and measurements are performed and only after a trial with an access client ended, it is executed the next, with another type of client. Such experiments are referenced as already described, according to the type of client, service and to computational operation implemented.

As for the Quality of Experience reviews present in the results, it is necessary to mention that such an assessment is subject to the perception of a group of evaluators, the authors of this study. Also, note that 
the execution of the experiments was recorded by a camera and then displayed to other members of the research group and all agreed with the assessment perceived by the authors (QoE).

\section{RESULTS}

\subsection{Video Service}

To consolidate the knowledge about the behavior of throughput in networks with desktop virtualization a relatively large amount of samples is required. Thus, 100 measurements of $60 \mathrm{sec}$ each were obtained for the video service analysis. As already mentioned above, a continuous video, without repetition of passages, with duration of more than one hundred minutes was used. The information obtained are only described graphically as follows. Figure 3 illustrates the results, while Table 1 shows the perceived QoE.

\subsection{Audio Service}

The tests for the audio service were performed identically to the last one, video service tests described above. So it also showed a significant number of samples and used the same equipment attached in the same way, changing only the service to audio.

The audio file used for playback had over $100 \mathrm{~min}$ in length and throughput measurements were obtained for each one-minute interval. This was performed for the first one hundred minutes (duration of test). The following refers to the average throughput for each time block of sixty seconds. Figure 4 and Table 2 show the results for the audio service.

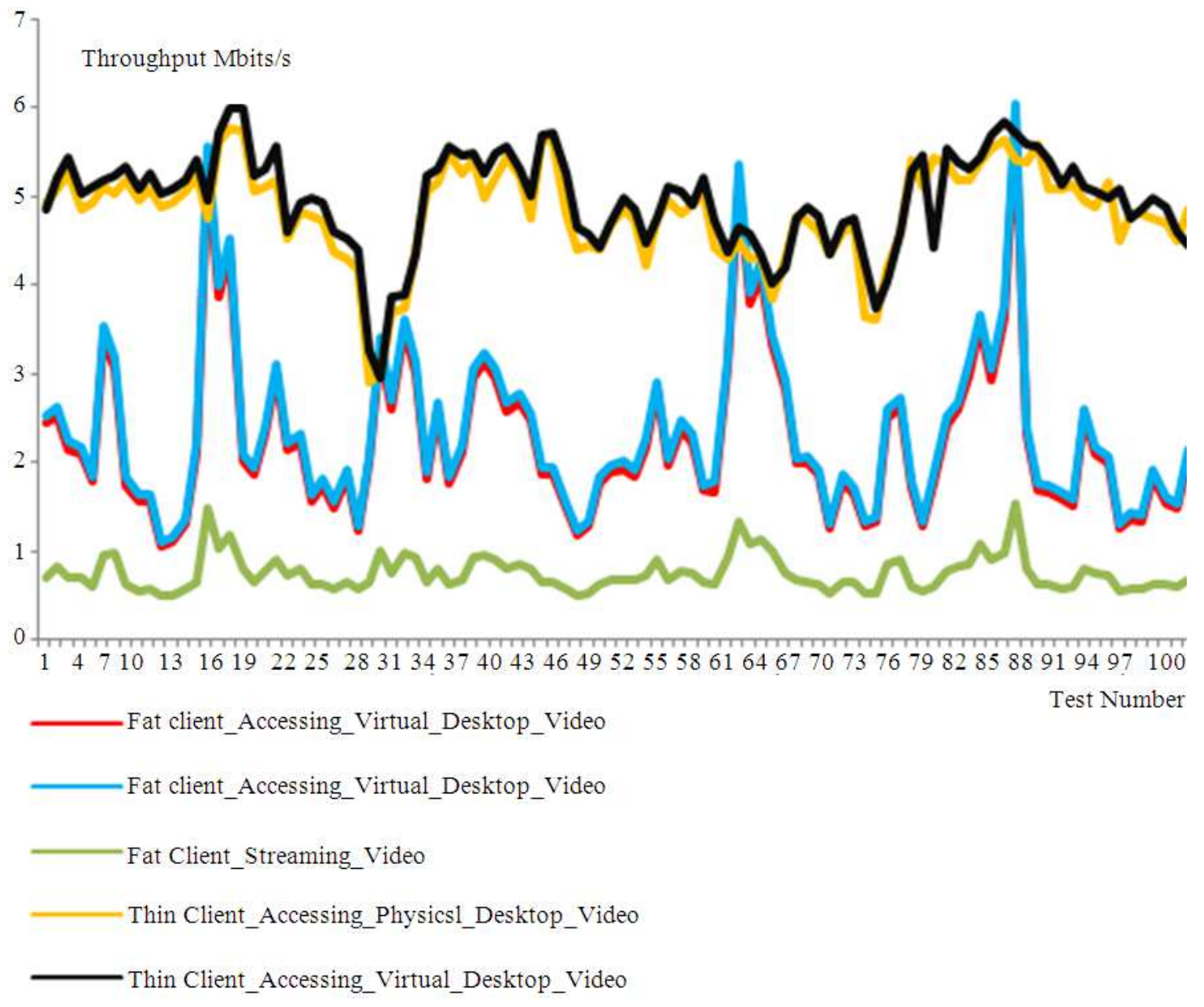

Fig. 3. Throughput Results for the video service 


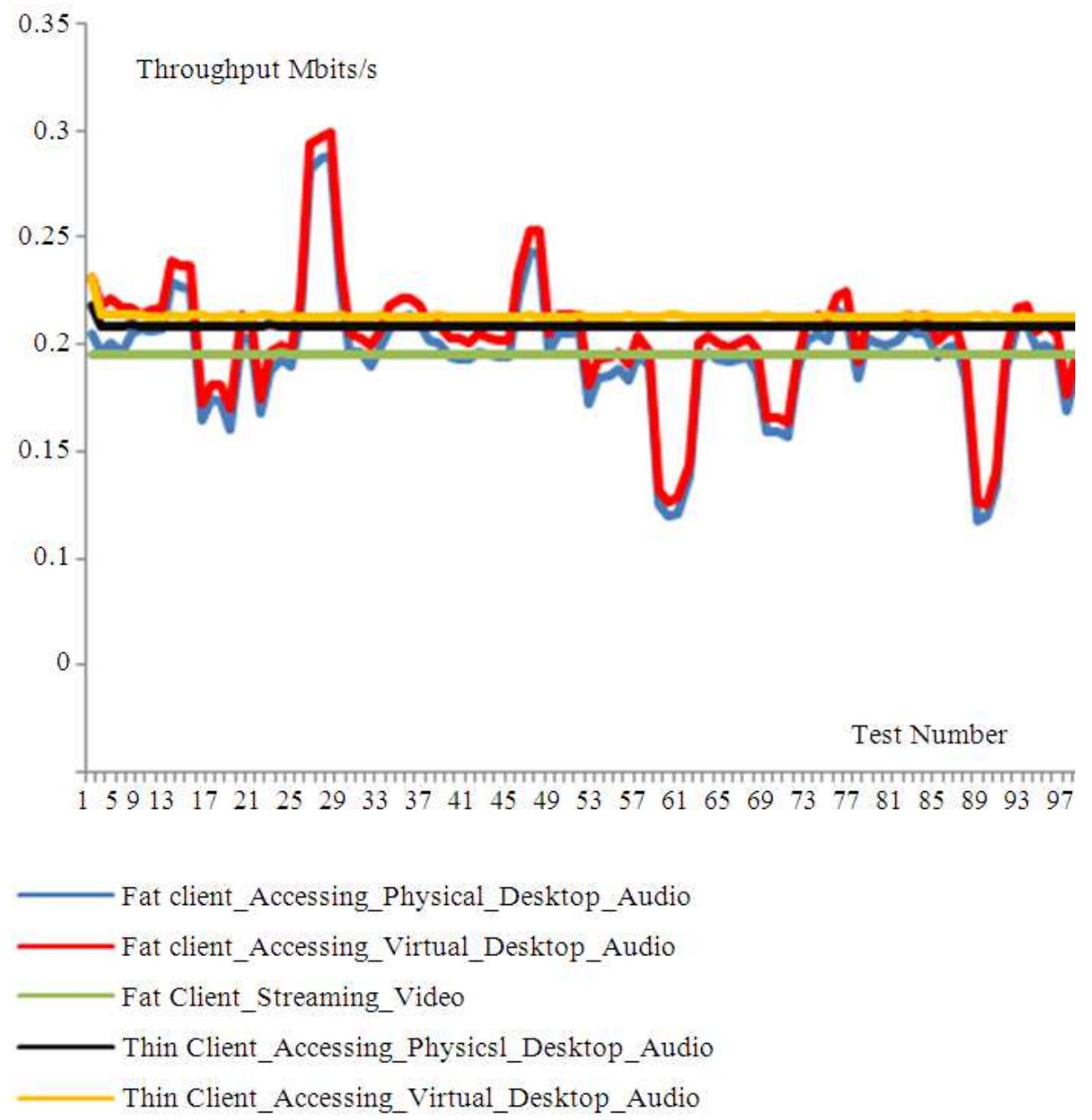

Fig. 4. Throughput results for the audio service

Table 1. Avarage throughput and QoE for the video service

\begin{tabular}{llll}
\hline Structures-setups & Throughput Mbits/s & Standard deviation & QoE \\
\hline Fat client_Accessing_Physical_Desktop_Video & 2,27530 & 0,0917 & Satisfactory \\
FatClient_Accessing_Virtual_Desktop_Video & 2,35739 & 0,0948 & Satisfactory \\
FatClient_Streaming_Video & 0,73757 & 0,0199 & Satisfactory \\
ThinClient_Accessing_Physical_Desktop_Video & 4,82345 & 0,0540 & Unsatisfactory \\
ThinClient_Accessing_Virtual_Desktop_Video & 4,95285 & 0,0546 & Unsatisfactory \\
\hline
\end{tabular}

Table 2. Avarage throughput and QoE for the audio service

\begin{tabular}{llll}
\hline Structures-Setups & Throughput Mbits/s & Standard deviation & QoE \\
\hline FatClient_Accessing_Physical_Desktop_Audio & 0,19504 & 0,00288989 & Satisfactory \\
FatClient_Accessing_Virtual_Desktop_Audio & 0,20418 & 0,00299489 & Satisfactory \\
FatClient_Streaming_Audio & 0,19520 & $4,4947 \mathrm{E}-05$ & Satisfactory \\
ThinClient_Accessing_Physical_Desktop_Audio & 0,20912 & $9,1320 \mathrm{E}-05$ & Satisfactory \\
ThinClient_Accessing_Virtual_Desktop_Audio & 0,21344 & 0,00018275 & Satisfactory \\
\hline
\end{tabular}




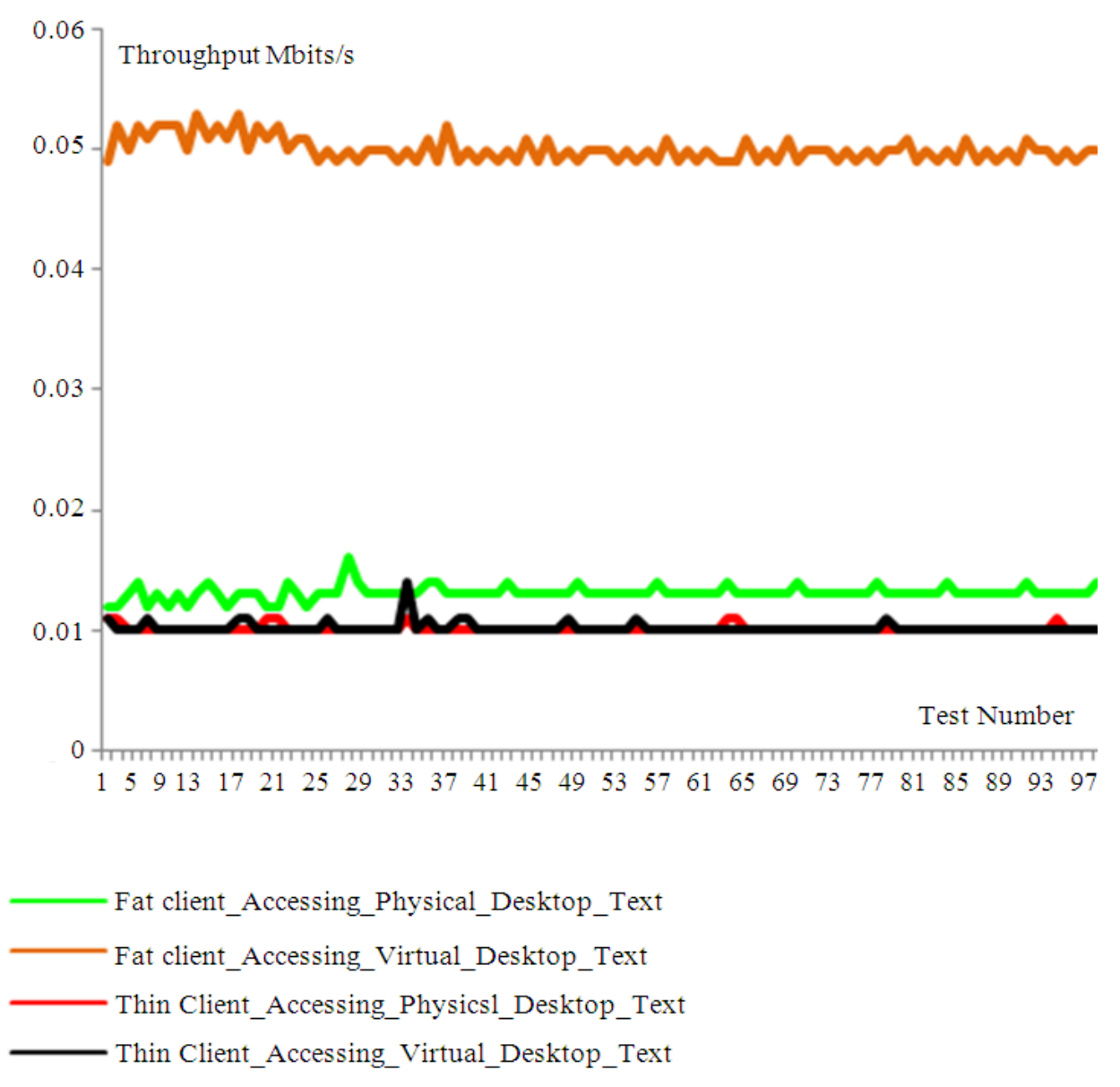

Fig. 5. Throughput results for the text service

\subsection{Text Service}

The Notepad tool specifies the text service tested in this study. Note that the streaming operation has no applicability for this type of service, since it is exclusively used for streaming media flow. Thus, there remained four experimental setups to observe the service studied. The Fig. 5 along with Table 3 illustrate throughput and QoE for this service.

It is important to keep inmind that the tests were $100 \mathrm{~min}$ long, segmented into a hundred equal measures. In addition, it's worth reminding that the same hardware equipment present at diagnosis for the audio service is employed.

\subsection{Navigation (Web Browser) Service}

To analyze the navigation service it was conducted two typification of tests. The first, lasting ten minutes, divided into measurements every sixty seconds and another one, of $10 \mathrm{~min}$ as well, but no subdivisions, obtaining conjugated ('integrated') results.

Verifying this service, the experiment availed itself of the same tooling of hardware and software present in the text evaluations. However, the text application was replaced by a software tool called Web browser. The operation, as previously described, aimed at accessing pages randomly, simulating the activity of a user's system. Figure 6 below reflects the throughput measurements obtained for the first type mentioned (subdivided) and displays them graphically. Figure 7 shows the bytes trafficked during the $600 \mathrm{sec}$ of each set for the second type (integrated). While Table 4 presents the perceptions regarding the quality of the experience (QoS), which applies to both classifications of experiments. 
Vitor Chaves De Oliveira et al. / Journal of Computer Science 9 (7): 827-837, 2013

Table 3. Avarage throughput and QoE for the text service

\begin{tabular}{llll}
\hline Structures-Setups & Throughput Mbits/s & Standard deviation & QoE \\
\hline FatClient_Accessing_Physical_Desktop_Text & 0,01309 & $5,70221 \mathrm{E}-05$ & Satisfactory \\
FatClient_Accessing_Virtual_Desktop_Text & 0,05003 & 0,000102942 & Satisfactory \\
ThinClient_Accessing_Physical_Desktop_Text & 0,01008 & $2,7266 \mathrm{E}-05$ & Satisfactory \\
ThinClient_Accessing_Virtual_Desktop_Text & 0,01015 & 0,000050 & Satisfactory \\
\hline
\end{tabular}

Table 4. Avarage throughput and QoE for the navigation service

\begin{tabular}{ll}
\hline Structures-Setups & QoE \\
\hline FatClient_Accessing_Physical_Desktop_Web & Satisfactory \\
FatClient_Accessing_Virtual_Desktop_Web & Satisfactory \\
ThinClient_Accessing_Physical_Desktop_Web & Satisfactory-with exceptions \\
ThinClient_Accessing_Virtual_Desktop_Web & Satisfactory-with exceptions \\
\hline
\end{tabular}

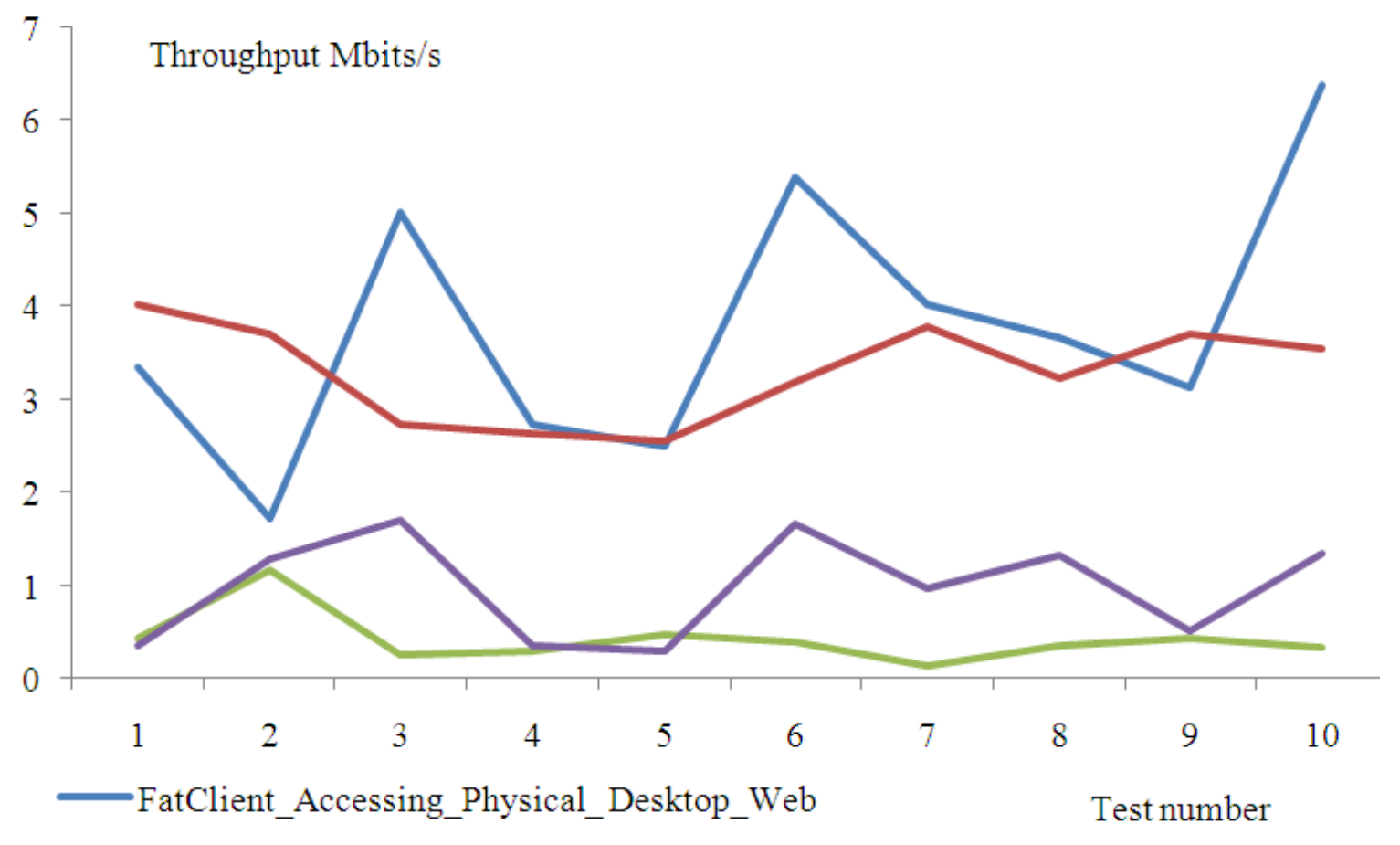

—FatClient_Accessing_Virtual_Desktop_Web

—ThinClient_Accessing_Physical_Desktop_Web

—ThinClient_Accessing_Virtual_Desktop_Web

Fig. 6. Throughput results for the navigation service (divided) 


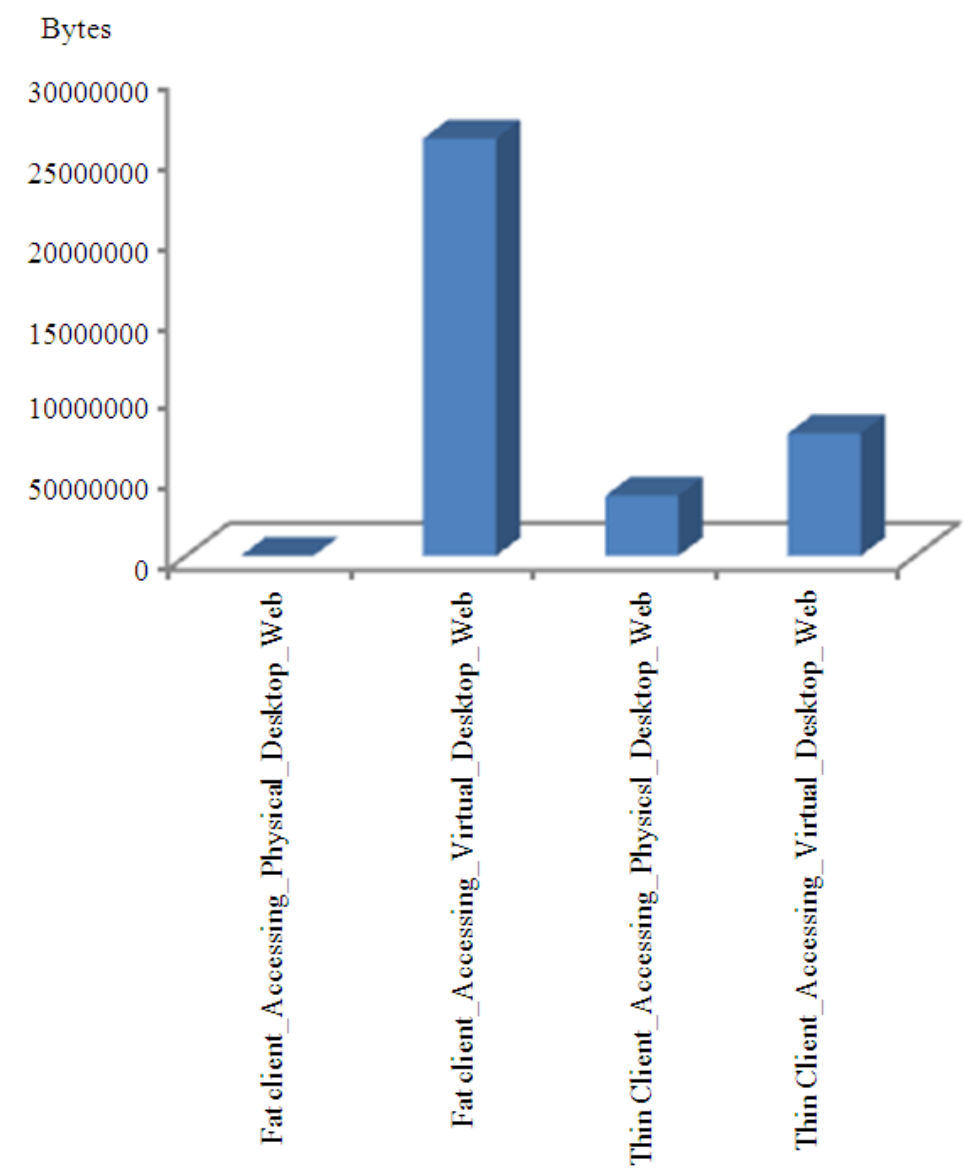

Fig. 7. Total Throughput Results for the navigation service (conjugated)

The thin client's performance in relation to the Quality of Experience, was satisfactory, but with exceptions. This is because it performed functional, but with scrolling slower than the Robust Client. In the tests conducted, it is clear the difference between accessing content via Web Browser between the two clients typifications. It was also observed that for pages with unique content in text Thin Client provides a Quality of Experience identical to the Fat Client and that this quality, in terms of loading time and to scroll the screen diverge away in accordance with the increasing amount of multimedia content and images on the web page.

\section{DISCUSSION}

The first service, video, showed the highest throughput demand in relation to the other services for all cases investigated. Another feature observed in the video was poor (low) quality of experience offered to the user at all times which used reduced hardware on the client. The comparison between the conventional structure and VDI showed that the cost of desktop virtualization is significant for video, since the average throughput in the case of using DV was more than triple that in the event that used the conventional desktop. Such behavior can be explained by the fact that the VDI is totally dependent on user interaction and it prevents the use of artifices that require less network QoS, such as buffering. Regarding the performance of the thin client for this application with VDI, it is noted that in addition to result in unsatisfactory $\mathrm{QoE}$, such employment required a throughput that, on average, was twice the required by the robust client. So, it's possible to objectively observe that is not advantageous, in relation to the network load, to employ reduced local hardware in the client access for the video service, otherwise it would 
add a high cost (in terms of throughput) to the network and still produce a quality of experience that does not meet the needs of this application.

The second service, audio, reproduced a sound with satisfactory QoE for all tests in all structures examined and presented a low throughput. This parameter, on average, showed minimal variations remaining constant during most of the tests. As for the reduction of the local hardware using the Thin Client it was considered as appropriate, once it resulted in a applicable performance compared to the robust client in all compositions analyzed. Thus, it is explained that the audio service when used in a desktop virtualization solution does not entail high costs to the network and allows variation in local client without harming the quality of experience.

The third service, text, followed the trends of the audio service, presenting an excellent QoE and a low throughput and it also supported the use of thin client in the access equipment role. The term "transparent" used to describe a behavior in which a change to another situation is not noticed can be applied in tests with this service. This is because both from physical to virtualized remote structure and from Thin Client to Fat Client there were no changes in the network behaviour or in the performance of this software tool. Therefore, this application instigates the use of VDI for various types of software products that deal primarily with text only. Some of those are known as ERP (e.g., SAP, DATASUL) and groupwares (e.g., ICQ, MSN, Outlook).

The forth service, navigation via Web Browser, outlined a differentiated framework for the QoE and throughput. The throughput varied according to the access client's type employed. The TC demanded about a third of the throughput presented by the robust client. For the performance analysis through QoE perception, the Fat Client showed a fully satisfactory scenario, 'transparent'. While for the Thin Client, there was something new, a kind of compromise in the subject of quality of performance experience. That is, during the investigations, page scrolling and page switching worked, but in longer periods of time compared to tests conducted with the robust client. In addition, it was noted that this additional time is reduced according to the decrease of graphical content on the page and, if the page does not contain such enrichment, the throughput behavior and QoE performance approaches the FC's. It is also pointed out that the performance of the $\mathrm{FC}$ allowed faster access to more information, culminating in greater network traffic communication. In view of these results, it is concluded that the navigation service consumes significant network throughput and that it is modified according to the amount and type of content accessed.

Furthermore, it shows that the use of reduced hardware on the client should be aligned to the type of content that will be accessed. That is, if the user needs to access applications that are textual, such as news, books and mail, the TC can be an interesting choice. But, if there is a need for graphical content such as images and videos, it is advisable to choose the robust client. Remember that, currently, a variety of software tools run on Web platform. So, if access via Thin Client is intended, designing a simple graphical ('plain text') user interface should be mandatory. This means that this evaluation can act as an indicator for the development of software applications, setting in the requirements analysis the VDI and the local hardware intended as the access client.

\section{CONCLUSION}

In view of the above, it is reiterated that this study sought to present the feasibility of VDI solutions in an expository way. This study has shown the cost of desktop virtualization in various local hardware environments and software applications, regarding the throughput load on the network. It sought to provide a background for a subject that is in the current corporate agenda and which knowledge its impacts are still uncertain. This research showed these effects for various common services to computer users and to various access clients. This made it possible to identify the feasibility of using VDI for different purposes, which is the main contribution of the research described.

For future work, it is interesting to evaluate, capture and analyze other quality of service parameters such as jitter, latency and losses. There is also the possibility to vary the type of channel, such as optical and wireless networks while increasing network complexity, subsequently increasing the number of elements in the network: clients, nodes and intermediate routers.

\section{REFERENCES}

Adibi, S., R. Jain, S. Parekh and M. Tofighbakhsh, 2010. Quality of service architectures for wireless networks: Performance metrics and management. 1st Edn., IGI Global snippet, USA., ISBN-10: 1615206809, pp: 691.

CITRIX, 2011. Desktop virtualization and security: A global market research report. Citrix Systems Inc. 
Eden, M., 2011. A Survey of Performance Modeling and Analysis Issues in Resource Management Across x86-based Hypervisors in Enterprise Cloud Computing Deployments.

GCNET, 2012. General Aspects in Data Networks (in portuguese). Computing Center-GCNET UNICAMP.

Mota, L.T.M., A.D.A. Mota and L.F. Fontolan, 2011. Quality of service policy for IEEE 802.11 networks with service rate selection based on fairness index. J. Comput. Sci., 7: 600-604. DOI: 10.3844/jessp.2011.600.604
Oliveira, V.C., A.A. Mota, L.T.M. Mota, 2013. Impacts of application usage and local hardware on the throughput of computer networks with desktop virtualization. Am. J. Applied Sci., 10: 117-122. DOI: 10.3844/ajassp.2013.117.122

Thibodeau, P., 2012. Server virtualization pushes storage demand to new highs. ComputerWorld. 